Research Article

\title{
Experimental Teaching of Information Security Based on Virtual Simulation
}

\author{
Xiaoqing Dai $\mathbb{1}^{1,2}$ \\ ${ }^{1}$ School of Computer Science, Chengdu Normal University, Chengdu 611130, Sichuan, China \\ ${ }^{2}$ Key Laboratory of Interior Layout Optimization and Security, Institutions of Higher Education of Sichuan Province, \\ Chengdu Normal University, Chengdu 611130, Sichuan, China
}

Correspondence should be addressed to Xiaoqing Dai; 990026@cdnu.edu.cn

Received 2 August 2021; Revised 2 November 2021; Accepted 10 November 2021; Published 28 November 2021

Academic Editor: Sang-Bing Tsai

Copyright ( $\odot 2021$ Xiaoqing Dai. This is an open access article distributed under the Creative Commons Attribution License, which permits unrestricted use, distribution, and reproduction in any medium, provided the original work is properly cited.

\begin{abstract}
In information security technology, virtual simulation technology has been applied in many fields, and its value has been proven and has attracted people's attention. Especially today, as a new type of computer model, virtual simulation covers the Internet and plays an important role in the information security research of big data platforms. This thesis aims to study and solve the problem of lack of experimental environment for information security technology in schools and to bring new teaching methods to the experimental teaching of information security technology in schools. At the same time, it emphasizes the teaching characteristics of the platform based on technology implementation and combines the cognition of high school students in the design and implementation of the platform. After understanding the main points, a research teaching design was carried out, which made up for the current virtual simulation experiment platform that pays attention to technology but despises teaching design. As a result of the empirical analysis, the virtual experiment simulation platform can solve the problems in the school's information security technology experiment teaching, increase students' interest in information security technology, and achieve the educational goal of cultivating students' independent innovation spirit and practical ability. From 2014 to 2016, the Ministry of Education started the construction of virtual simulation information security experiment teaching. Currently, 290 virtual simulation information security experiment teaching has been implemented and distributed in 26 provinces and cities, of which 199 are engineering, 81 are finance, and 10 are education.
\end{abstract}

\section{Introduction}

1.1. Background of Topic Selection. In recent years, information security has become a concern of people, and the market demand for information security management and development talents is also growing. But now, information security talents are still scarce. Most of the information security talents are in universities and research institutions and rarely in the economic market. According to the current development of information technology, the society's demand for professionals in the information security field reaches hundreds of thousands. In order to solve this contradiction, it is necessary to speed up the cultivation of information security talents. However, since our country's information security teaching has only recently developed, there must be insufficient talent training. At this time, information security experiment teaching based on virtual simulation can just meet this need. The research can demonstrate information securityrelated technologies. It also allows students to use and configure information security products, such as real simulated encryption software, network attacks, and network firewall and intrusion detection system so that students can gain relevant knowledge and practical experience in this virtual laboratory environment.

1.2. Significance of the Research. Virtual simulation of information security experimental teaching has brought new teaching methods and learning methods to school information technology experimental teaching and has played a role in promoting the development and implementation of high school information technology experimental courses. 
In addition, the implementation of virtual simulation speeds up the process of information security education, making electronic resources (such as experimental virtual simulation systems) more important in future experimental teaching. In order to improve the quality of teaching, firewalls, network monitoring, and other subsystems related to information security experiments have been developed. In such teaching courses, students can not only really play a role, but they can also simulate building offensive and defensive networks and write their own programs. Teachers only need to provide students with basic source programs in the form of classes, modules, or components to assist. Therefore, students have a deeper understanding of the design and implementation technology of the aforementioned system.

\subsection{Related Work of Information Security Experimental} Teaching Research Based on Virtual Simulation. Rosedale believes that under the virtual simulation technology, information protection is very important. Whether it is a government agency or a commercial organization, it must have a corresponding use policy when using consent information [1]. Bastug et al. pointed out that in order to ensure data security, the "Cyber Security Law" must be strictly enforced, which is the most important [2]. In addition, it is necessary to improve system specifications, study and formulate relevant laws and regulations, establish support systems, and strengthen the design, development, promotion, and implementation of network security and construction work. Riva et al. believes that China has many information security regulations but no regulations [3]. This kind of nonstandard and lack of systematic system is difficult to guarantee information security. Therefore, it is urgent to speed up the legislative process of the Information Security Law. However, we think that due to the limitation of disciplines, the lack of research on virtual simulation technology will inevitably lead to the inability to understand the root cause of information security problems.

\subsection{Innovation Points of This Research.}

(1) The virtual simulation method is used in the research of information security experiment teaching [4].

(2) The use of virtual simulation can vividly simulate the operation of the experimental process. The vivid learning environment enables students to obtain intuitive knowledge and improve their understanding of abstract principles. To achieve the goal of cultivating high-quality talents with less time and less cost, it can also be combined to evaluate and improve teaching effects. The virtual simulation training system can evaluate knowledge points, operating points, and experimental procedures for simulation training [5].

\section{Methods of Information Security Experimental Teaching Research}

2.1. Concept of Information Security. Information security means that the hardware and software of the information network and the data in the system are protected from accidental or malicious reasons from being damaged, altered, or leaked, the system operates continuously, reliably, and normally, and the information service is uninterrupted. Information security mainly includes the following five aspects, namely, confidentiality, authenticity, integrity, noncopyability, and security. Network and data in the computer information system are protected from damage, alteration, or leakage due to accidental or malicious reasons, and the system is always reliable and safe [6]. It runs continuously without interrupting information services.

Information security itself is a broad and abstract concept. Generally speaking, the focus of information security is the security of the information itself. Its task is to protect information assets, prevent information from being leaked, tampered, destroyed, undeniable, etc., so that it will not cause loss while maximizing the use of information. For the definition of information security, there is no uniform definition in the international scope. Information system refers to the protection of the system to ensure that it provides stable services. The results of information security have been widely recognized through the application of operating system security and system information security. It generally refers to the security of information technology systems or the security of a certain information system (such as banking information systems, national defense security systems, and so on). From its original and technical point of view, information security generally refers to the protection of information systems (including hardware, software, data, personnel, physical environment, and infrastructure), to prevent the system from being attacked and leaked and to ensure that the information system can operate normally without being terminated and that the services provided can be reliably guaranteed. Nowadays, from the perspective of information network systems, modern information security is widely considered to include the following meanings:

(1) The security of the operating system includes the following aspects: the scientific management of the information network system; the establishment, implementation, and improvement of rules and regulations; the responsibility, vigilance, and foresight of management personnel; protection of laws and policies (such as whether users have legal rights and whether policies are allowed); physical control security; hardware operation security; operating system security (such as whether data files can be protected); avoid and eliminate deadlocks; and prevent electronics information leakage.

(2) System information security includes user password authentication, user access authority control, data access authority and audit, data encryption, etc. This article is based on the information assets of the enterprise, such as data, information, and so on. Regarding the security of the operating system, we can also guarantee by using methods such as 
strengthening passwords, doing a good job of border defense, updating software, closing unused services, and using data encryption.

Figure 1 shows the relationship between information security requirements and security threats.

\subsection{Features of Information Security}

2.2.1. Confidentiality. Confidentiality means that information provided to unauthorized individuals or entities in order to prevent information from being leaked out is provided only for authorized users [7].

2.2.2. Completeness. Integrity means that in the process of storage or transmission, information may not be accidentally or intentionally deleted, modified, forged, interfered, copied, inserted, damaged, or lost [8].

2.2.3. Authenticity. Authenticity means that all participants are not allowed to refuse or deny the tasks and promises they have performed according to law [9].

2.2.4. Availability. Availability means that information can be accessed by authorized institutions and used on demand [10].

2.2.5. Controllability. Controllability means that authorized institutions can control information at any time.

Among the five major characteristics of information security, integrity and confidentiality are considered an essential function, integrity of information is a prerequisite for availability, controllability reflects confidentiality, and non-repudiation is included in integrity.

2.3. Key Technology of Information Security. The core issue of information security in modern information systems is cryptographic theory and its applications, and its foundation is the structure and evaluation of trusted information systems. Information security technology mainly includes the following:

(1) Host control technology

(2) Identity authentication technology

(3) Access control technology

(4) Information encryption technology

(5) Firewall technology

(6) Anti-virus technology

(7) Intrusion detection technology

(8) Security audit technology

The key technologies of information security are shown in Figure 2.

\subsection{Information Security-Related Theories}

2.4.1. HTP Theory. The HTP theoretical model was proposed by domestic information security experts Chen Wei and Sun Qiang. This model introduces the idea of "the wooden barrel principle" in management, that is, "a wooden barrel" is composed of many wooden boards. These boards are different in length, so the maximum capacity of the barrel does not depend on the long board but on the shortest board. This principle also applies to the field of information security. The model is mainly composed of three elements: personnel management, technical means and safety products, and activity process and overall framework. In the HTP theoretical model, it is emphasized that personnel management is the most important factor for information security. The quality of personnel organization and management is directly related to the success or failure of the security line of defense.

Compared with other theories, the HTP theory makes us realize that in a horizontal state, the long board is useless, and the water interface is in level with the shortest board.

2.4.2. Risk Management Theory with Risk Control as the Core. Risk management refers to the management process of how to minimize the risks of information systems in an environment that is definitely risky. It is a management method that uses the identification, measurement, and analysis of risks to choose the most effective way to deal with or eliminate risks proactively, purposefully, and in a planned way, and strive to obtain maximum security at the lowest cost. As a management activity of enterprises, risk management originated in the United States. In the 1960s, risk management theory was also applied to the field of information security by the United States for the first time. The goal of an organization's risk management of information security is to obtain the greatest security guarantee at the lowest cost. Around this goal, the organization should periodically identify information security risks, assess the risks and discover potential security risks, and take corresponding measures to deal with the risks. Therefore, information security management is a process of making security decisions around information security risk control. This process adopts appropriate risk control strategies to reduce risks to the acceptable range of the information system.

2.5. Virtual Reality. The real-time nature of the virtual simulation system requires that the system be able to quickly handle various response messages. It is one of the most prominent characteristics of virtual simulation that people can experience, and it is also a key factor to improve the harmony of human-computer interaction. The real-time research of virtual simulation has always been a key and hot issue in the field of virtual reality research. Real-time generation and dynamic display of scenes are important contents of the real-time research of virtual simulation. The traditional concept believes that scene modeling is the premise of real-time generation and real-time dynamic display of virtual scenes. In this regard, researchers have 


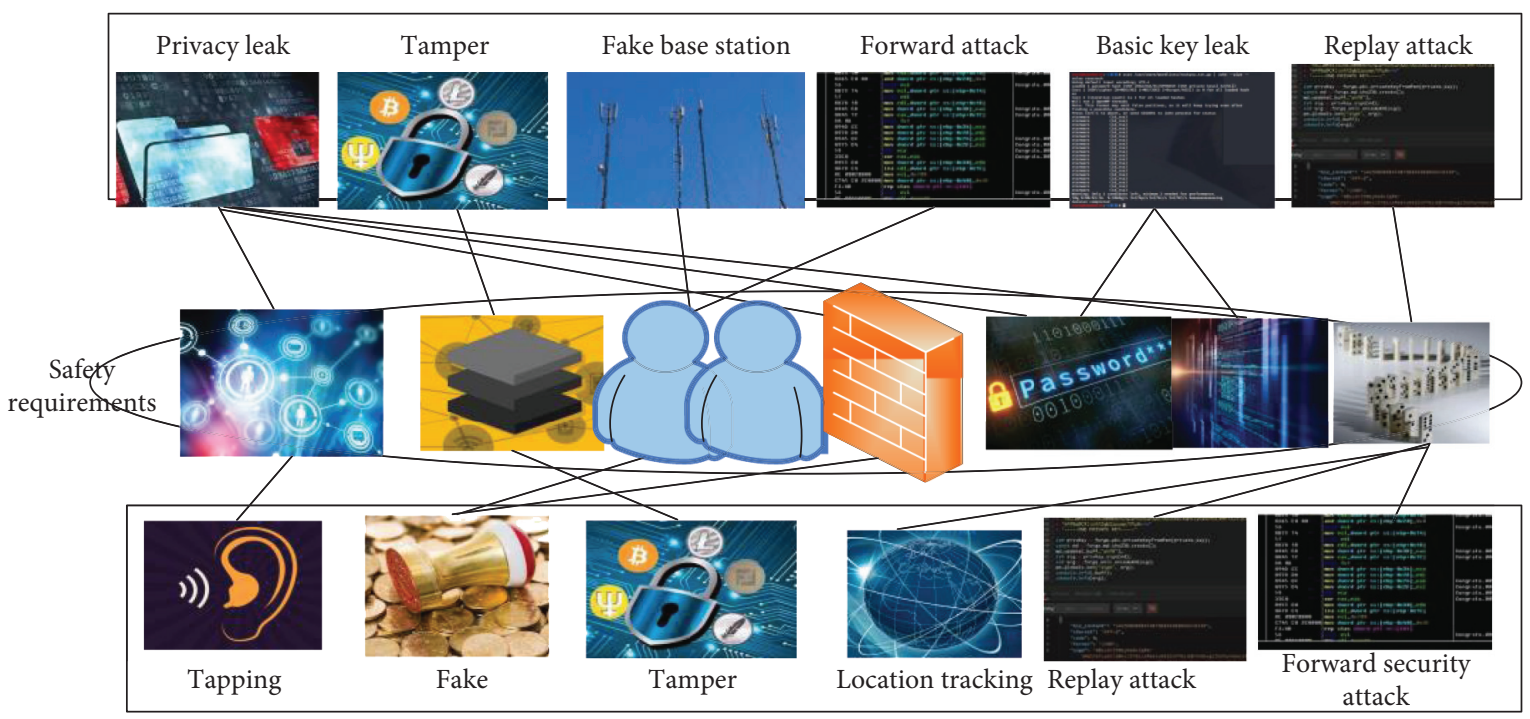

FIGURE 1: The relationship between system security requirements and security threats.

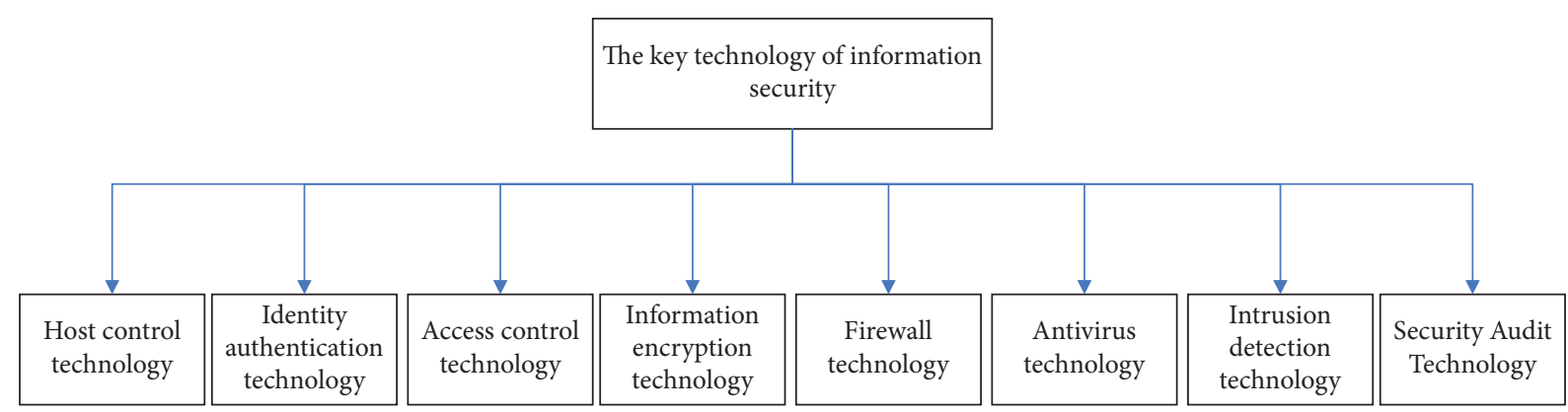

FIGURE 2: The key technology of information security.

been committed to improving the algorithm of model establishment to reduce the complexity of the model or minimize the impact on virtual simulation. There are many main solutions for small and inconspicuous operations. Among them, the most widely used is the multiprecision representation technology of the model. The basic idea of this method is to accurately model the observer's model part, and the other parts are simplified according to the importance. Figure 3 shows a frame diagram of the simulation rendering process.

The simulation rendering process includes message processing, rendering simulation, scene object, reply, initialization feature, and other related processes. These processes are interconnected during the simulation process and are indispensable. Among them, message processing is the most critical part of the entire simulation process. One link determines the success of the whole process.

In the development process of virtual reality technology, with the different actual needs in various aspects, it has gradually evolved into augmented virtual reality, immersive virtual reality, desktop virtual reality, and distributed virtual reality. Augmented virtual reality is a technology that integrates computer-generated content into the display of real videos in real time. It mainly refers to the creation of a realworld-oriented and annotated composite scene based on the application of various hardware technologies, which can then be passed. Image processing and computer vision technology can be used to achieve the purpose of realistic interaction between computer-generated elements and real image content. Immersive virtual reality refers to the technology that uses hardware devices such as headmounted displays, data gloves, and voice recognizers to participate in an immersive virtual reality system. It mainly closes the user's visual, auditory, and tactile channels so that the user can get a completely immersive experience. Generally speaking, this type of system will be equipped with head, hand, and eye direction trackers to track changes in the human body's senses and then achieve the real-time performance of the system. But this kind of application is not popular and is just used in some key experiments such as aerospace simulation. Desktop virtual reality refers to a system that implements simulation based on ordinary PCs or workstations. It mainly uses low-end graphics workstations to generate immersive three-dimensional effect scenes, so that users can observe the virtual reality through the display of ordinary PCs and use peripherals such as keyboard and mouse to interact with the virtual scene that the user sees. Desktop virtual reality is usually applied in lowcost systems, such as some education and training systems. Distributed virtual reality is a kind of virtual environment 


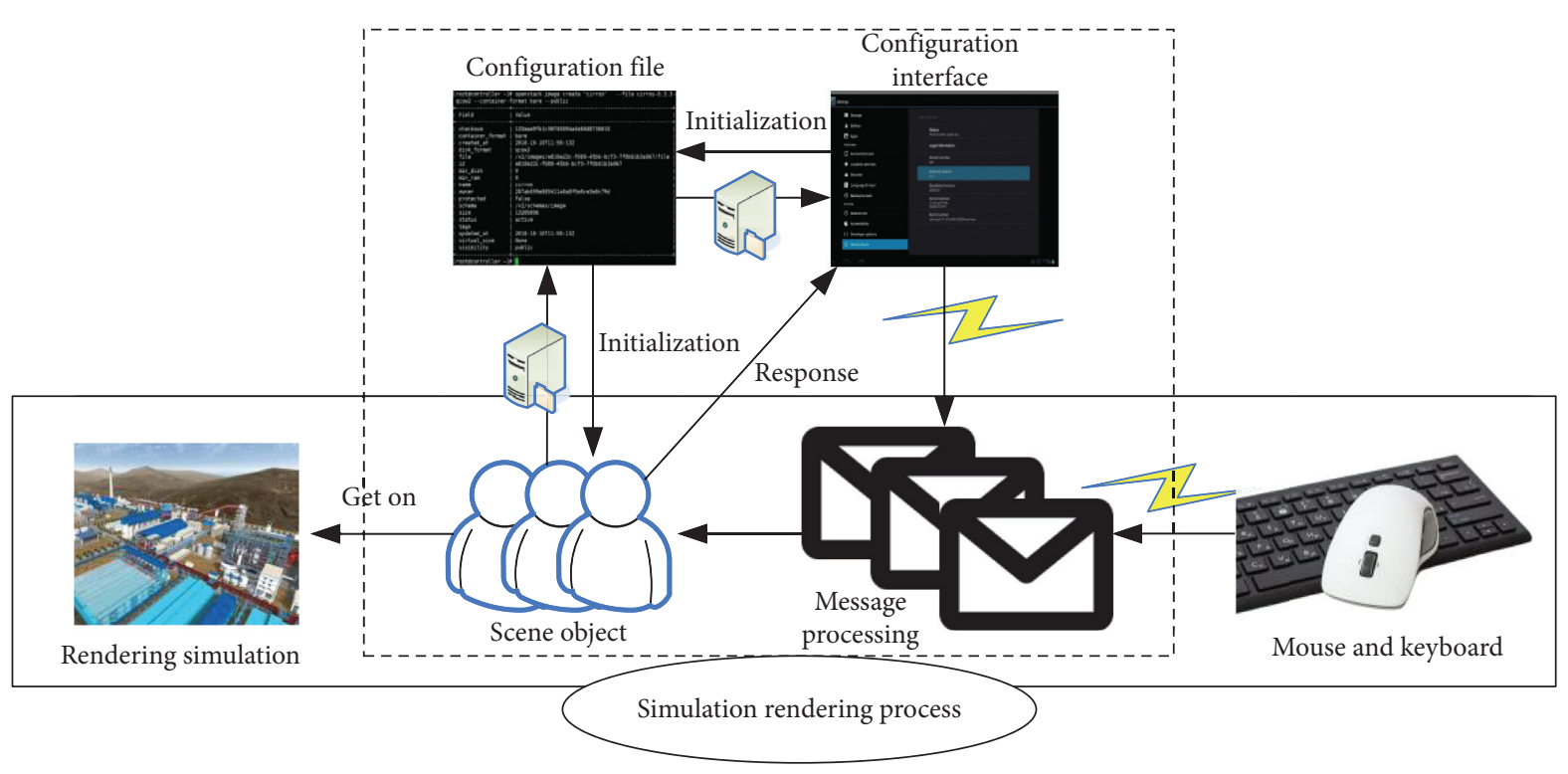

FIGURE 3: Simulation rendering process.

based on the Internet. It uses the Internet to associate multiple users in different physical locations together and exchange messages, so as to achieve the purpose of collaborative work between users. In this network-based virtual environment, users can communicate with other users conveniently without geographical restrictions. Today, with the rapid development of the Internet, distributed virtual reality has been widely used in education, engineering, commerce, and entertainment.

\subsection{Necessity of Constructing Virtual Simulation Information} Security Experiment Teaching. At this stage, whether it is a middle school or a university, experimental classes are indispensable. The virtual simulation experiment in the field of education and teaching has made up for many shortcomings of the current reality experiment to a large extent. It is a new type of experimental teaching method that breaks the single traditional teaching mode. Many school courses, including physics, chemistry, biology, medicine, and even sports kinesiology, can use virtual simulation experiments. This type of virtual simulation experiment is close to the teaching syllabus, has clear teaching goals, and is based on relevant education and teaching theories. It has good interaction performance and can cultivate students' creativity and independent thinking ability and stimulate their innovative consciousness. Virtual simulation experiments are mainly used for scientific research. Such simulation experiments often require more professional scientific theories as support and need to establish corresponding models. This type of model is generally a mathematical model or a mathematicalphysical effect model. The virtual simulation system uses this model to analyze and research the experimental results with the help of expert experience knowledge, statistical data, and information materials. In the process of experimental research, researchers put forward ideas, then set relevant parameters, create corresponding scenes, put ideas into experiments, observe phenomena, and finally obtain data and results and use relevant scientific methods for analysis and evaluation. For example, scientists at Cornell University in the United States used the world's most powerful computer cluster to model and simulate the state of motion of a small moon-shaped object on Jupiter 1 billion years ago, thereby, revealing the mystery of its unusual trajectory. In addition, there is a virtual simulation system of car collision used in the study of car collision science to study the collision behavior and safety performance of different models of cars in different situations, saving financial and material resources. Its necessity can be divided into the following points:

(1) Experiment in a real experimental environment, which is very destructive: due to the destructive nature of network information security, as well as offensive and defensive technologies, creating network security loopholes in teaching will constitute a huge risk [8]. Experiments such as network attacks and virus injections in a real network environment will lead to disastrous consequences. This makes this type of experimental teaching be based on virtual simulation technology [11].

(2) It is very complicated to create a real experimental environment, and the experiment cost is very high [12].

(3) Advantages of using "virtual simulation" to conduct information security experiments: since all the experimental processes are executed in a virtual way, students can log in remotely to participate in the entire experimental project, set up the experimental environment flexibly and conveniently, and quickly restore the experimental environment [13]. On the premise of achieving the same educational effect, the destructiveness of real experiments is largely reduced [14]. 


\section{Experiments on Information Security Experimental Teaching Research}

3.1. Information Security Experiment Platform Architecture Based on Virtual Simulation. The system architecture of the simulation experiment platform includes control servers, network devices, virtual networks, cloud storage, virtual machine image libraries, etc., as shown in Figure 4.

3.2. Three-Dimensional Model Conversion Theory. In the virtual scene, the attributes of the $3 \mathrm{D}$ model (such as position, size, and rotation) are transformed based on the mathematical model [15]. The three-dimensional model conversion can be regarded as the same coordinate system, the model does not change or the model does not change, the coordinate system does not change, and the latter usually uses more of the former. The essence of three-dimensional model conversion is that the computer performs linear equation operations, and the basic calculation formula is as follows:

$$
N_{u}=\left[\begin{array}{llll}
n_{00} & n_{01} & n_{02} & n_{03} \\
n_{10} & n_{11} & n_{12} & n_{13} \\
n_{20} & n_{21} & n_{22} & n_{23} \\
n_{30} & n_{31} & n_{32} & n_{33}
\end{array}\right]\left[\begin{array}{l}
u_{0} \\
u_{1} \\
u_{2} \\
u_{3}
\end{array}\right] .
$$

After completing the specified operation, the computer performs matrix operations on the data of the three-dimensional model to obtain new data of the three-dimensional model and realizes the transformation, rotation, and scaling transformation of the three-dimensional graphics [16].

3.2.1. Graphic Translation Transformation. Translation refers to moving all points on a graph in the same plane along a certain straight line direction for the same distance. The translation matrix is expressed in the following equation:

$$
T\left(t_{x}, t_{y}, t_{z}\right)=\left[\begin{array}{cccc}
1 & 0 & 0 & t_{x} \\
0 & 1 & 0 & t_{y} \\
0 & 0 & 1 & t_{z} \\
0 & 0 & 0 & 1
\end{array}\right] .
$$

3.2.2. Graphic Rotation Transformation. The rotation matrix is a matrix obtained by rotating each vertex of the object by a certain angle with a straight line passing through the origin as a rotation axis. Rotation will change the vertex direction and face normal of the object, but it will not change the face information [17]. The three-dimensional graphics rotate around the $x$-axis, $y$-axis, and $z$-axis, and the rotation matrix is, respectively,

$$
\begin{aligned}
& R_{x}(\theta)=\left[\begin{array}{cccc}
1 & 0 & 0 & 0 \\
0 & \cos \theta & -\sin \theta & 0 \\
0 & \sin \theta & \cos \theta & 0 \\
0 & 0 & 0 & 1
\end{array}\right], \\
& R_{y}(\theta)=\left[\begin{array}{cccc}
\cos \theta & 0 & \sin \theta & 0 \\
0 & 1 & 0 & 0 \\
-\sin \theta & 0 & \cos \theta & 0 \\
0 & 0 & 0 & 1
\end{array}\right], \\
& R_{z}(\theta)=\left[\begin{array}{cccc}
\cos \theta & -\sin \theta & 0 & 0 \\
\sin \theta & \cos \theta & 0 & 0 \\
0 & 0 & 1 & 0 \\
0 & 0 & 0 & 1
\end{array}\right] .
\end{aligned}
$$

Based formulas (3)-(5), a transformation matrix rotating around any axis can be obtained. Assuming that the arbitrary axis is a straight line passing the point $(x, y, z)$ and the direction is $(a, b, c)$, the rotation angle is $\theta$, and the reverse derivation of (5)-(3) can be obtained [18]. The total transformation matrix is

$$
N\left(\left[\begin{array}{l}
x \\
y \\
z
\end{array}\right]\left[\begin{array}{l}
a \\
b \\
c
\end{array}\right] \theta\right)=T(x, y, z) R_{x}(\alpha) R_{y}(\beta) R_{z}(\theta) R_{y}(-\beta) R_{x}(-\alpha) T(-x,-y,-z)
$$

3.2.3. Graphic Scaling and Transformation. Scaling refers to reducing or enlarging an object by a certain ratio. Scaling transformation will change the shape of the object [17].
Usually, the scaling matrix is also expressed as a matrix formula of three scaling factors relative to the three coordinate axes, as shown in the following equation: 


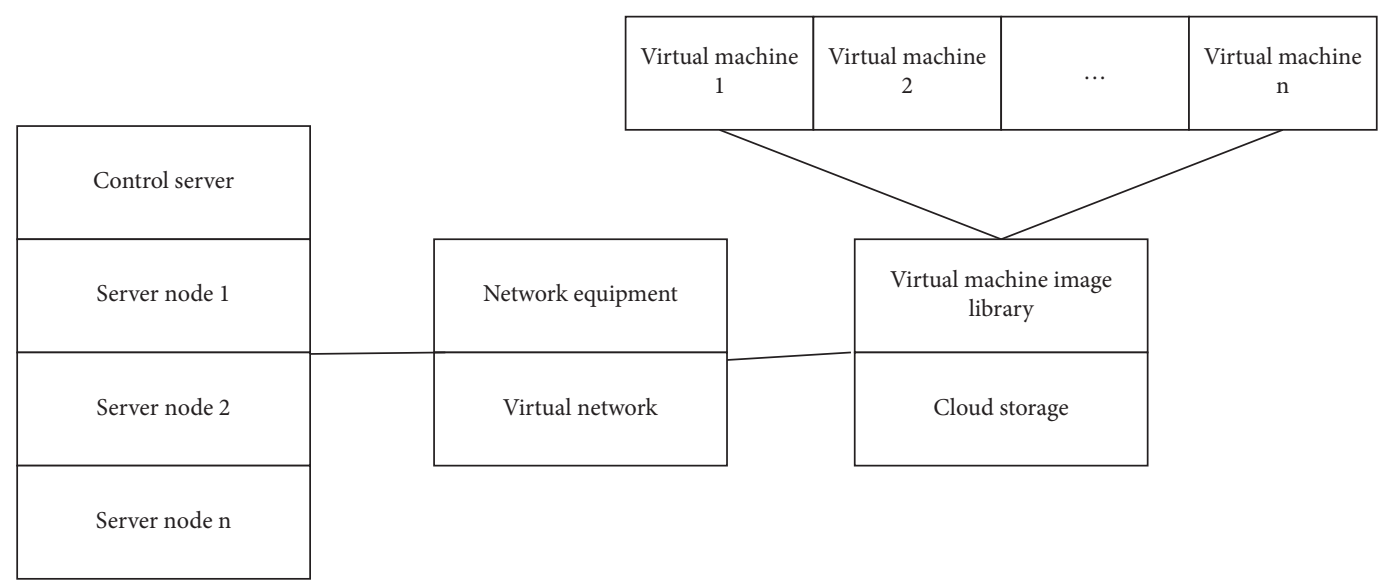

FIGURE 4: System architecture of network information security virtual simulation experiment platform.

$$
S\left(s_{x}, s_{y}, s_{z}\right)=\left[\begin{array}{cccc}
s_{x} & 0 & 0 & 0 \\
0 & s_{y} & 0 & 0 \\
0 & 0 & s_{z} & 0 \\
0 & 0 & 0 & 1
\end{array}\right] .
$$

\section{Information Security Experimental Teaching Research}

\subsection{Significance Analysis of Virtual Simulation Applied to Safety Information Experiment Teaching.}

(1) Virtual simulation creates a new learning scene:

Virtual simulation technology can create a virtual production environment, and the students look like they are on the manufacturing site. The manufacturing process is displayed on the screen, and students can communicate with them freely. The combination of virtual simulation technology and network technology can provide students with an open and brand-new teaching environment [19].

(2) Virtual simulation provides a new teaching method:

The use of virtual simulation technology can simulate various models required in the learning process or make virtual objects real, and students can intuitively observe the final results or effects in the virtual system. The virtual simulation system can virtualize virtual objects, create virtual classrooms, and virtualize different roles. Students can interact with virtual classmates and teachers in a virtual classroom atmosphere and carry out heuristic teaching [20].

(3) Virtual simulation has changed the traditional learning method:

Through virtual simulation technology, the limitations of printed books can be overcome, and open exploration and learning can be carried out [21]. Students can freely organize their own learning time, progress, methods, etc. Students can use the Internet to find suitable learning resources according to their own hobbies and specific situations, which is conducive to stimulating students' creative thinking and helping them to actively explore knowledge.

(4) Virtual simulation enriches experimental teaching content:

Through virtual simulation technology, some abstract, complex phenomena and processes that are difficult to observe directly can be correctly demonstrated [2]. Computer multimedia technology can be used to make simulation software and create virtual scenes, so that students can be immersed in the experiment process, so as to produce a better learning experience. It can also show the teaching content to students from all angles and directions, and the content of classroom teaching is also richer.

(5) Virtual simulation technology saves limited teaching costs:

This technology can be used for a variety of teaching tasks without the need to purchase a variety of actual learning and training equipment, which can save a lot of experimental supplies and reduce the loss of experimental equipment, thereby saving a lot of equipment costs [4].

\subsection{Survey Data Analysis}

4.2.1. Ability Training Situation after Using Virtual Simulation Platform. The results showed that among the 300 respondents, $211(70.3 \%)$ believed that their hands-on skills had been improved, 152 (50.7\%) believed that their ability to analyze problems had been improved, and 98 (32.7)\%) believed that their collaboration and communication skills have been improved. 266 (88.7\%) believed that their innovative design capabilities have been improved, and 177 (59.0\%) believed that their problem-solving skills have been improved.

The situation of ability training after using and not using the virtual simulation platform is shown in Figure 5. 


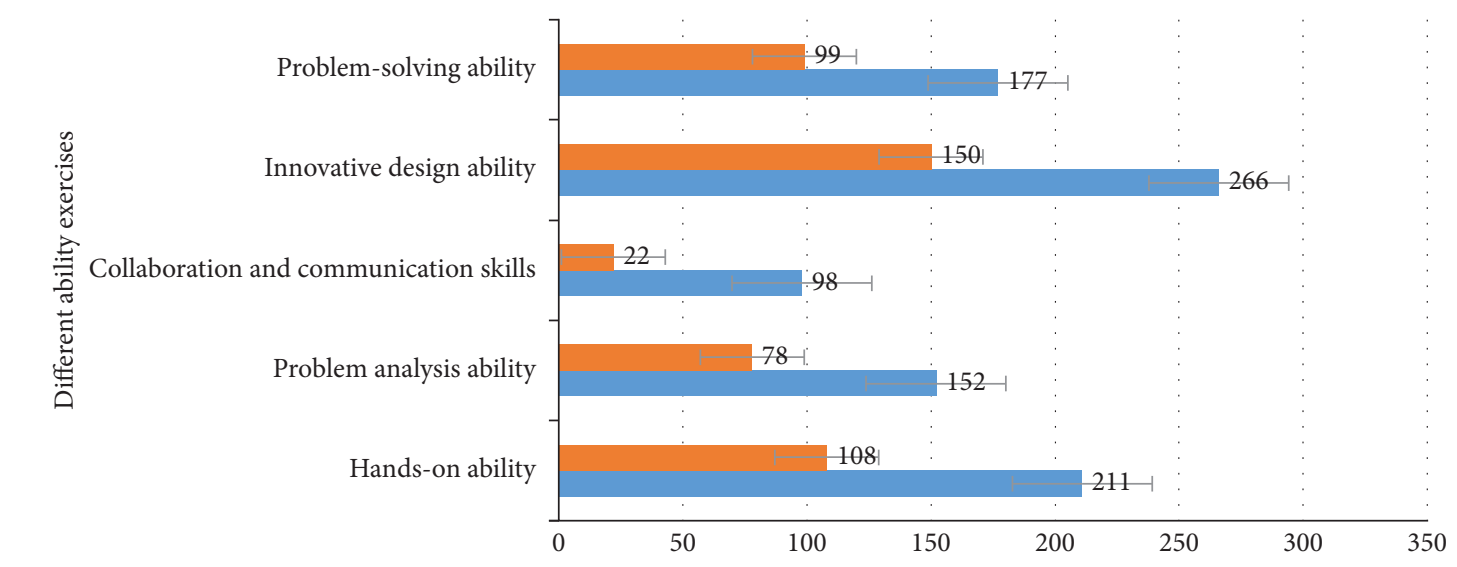

\begin{tabular}{|l|c|c|c|c|c|}
\cline { 2 - 5 } \multicolumn{1}{c|}{} & Hands-on ability & $\begin{array}{c}\text { Problem analysis } \\
\text { ability }\end{array}$ & $\begin{array}{c}\text { Collaboration and } \\
\text { communication } \\
\text { skills }\end{array}$ & $\begin{array}{c}\text { Innovative design } \\
\text { ability }\end{array}$ & $\begin{array}{c}\text { Problem-solving } \\
\text { ability }\end{array}$ \\
\hline a Never used & 108 & 78 & 22 & 150 & 99 \\
\hline Used & 211 & 152 & 98 & 266 & 177 \\
\hline
\end{tabular}

Number of people

never used

Used

FIgURE 5: Ability training situation after using and not using virtual simulation platform.

TABLE 1: Reasons that affect the application effect of virtual simulation experiment platform.

\begin{tabular}{lccccc}
\hline Reason & $\begin{array}{c}\text { Getting started is } \\
\text { difficult }\end{array}$ & $\begin{array}{c}\text { The operation process is not } \\
\text { realistic enough }\end{array}$ & $\begin{array}{c}\text { The operation process is } \\
\text { more complicated }\end{array}$ & $\begin{array}{c}\text { Cannot communicate } \\
\text { with peers }\end{array}$ & $\begin{array}{c}\text { Few learning } \\
\text { resources }\end{array}$ \\
\hline $\begin{array}{l}\text { Number of } \\
\text { people }\end{array}$ & 6 & 17 & 20 & 43 & 14 \\
Percentage & $6.0 \%$ & $17.0 \%$ & $20.0 \%$ & $43.0 \%$ & $14.0 \%$ \\
\hline
\end{tabular}

4.2.2. Reasons That Affect the Application Effect of the Virtual Simulation Experiment Platform. The results showed that among the 100 respondents, $6(6.0 \%)$ thought that the entry operation would hardly affect the application effect, 17 (17.0\%) thought that the operation process was not realistic enough to affect the application effect, 20 persons (20.0\%) thought that the experiment content is not comprehensive enough to affect the application effect, 43 people $(43.0 \%)$ thought that the inability to communicate with peers will affect the application effect, and 14 people (14.0\%) thought that the lack of learning resources will affect the application effect.

The reasons that affect the application effect of the virtual simulation experiment platform are shown in Table 1.

The reasons that affect virtual simulation include unrealistic operating procedures, inability to communicate with peers, and few learning resources. To reduce the impact, you can also improve operating proficiency, such as strengthening communication and providing more learning resources.

\section{Conclusion}

Network security is one of the important research contents in information security, and it is also a research hotspot in the current information security field. The research content includes the design and analysis of overall network security solutions, the research and development of network security products, etc. Network security includes physical security and logical security. Physical security refers to the physical protection of communications, computer equipment, and related facilities in the network system from damage and loss. Logical security includes information integrity, confidentiality, non-repudiation, and availability. It is a matter involving network, operating system, database, application system, personnel management, etc., which must be considered comprehensively.

The implementation of virtual simulation technology has played an important role in promoting courses related to information security experimental teaching. This technology not only enriches teaching content but also reduces teaching costs, overcomes time and space constraints, and moves the laboratory to the Internet. The most important thing is that it promotes students' enthusiasm for experimental teaching, can also motivate students' learning enthusiasm and initiative, can improve learning effects, and solve problems that are difficult to complete in school education. It can be said that the implementation of virtual simulation technology plays an important role in improving the teaching quality of network information security-related courses. Virtual 
simulation is the creation of a virtual environment that presents us the feeling in the way we feel it, as if we were really there. It uses a variety of technologies to achieve this goal and is a technologically complex feat that must take our perception and cognition into consideration. It is both entertaining and serious. The technology is becoming cheaper and more popular. It is foreseeable that due to the possibility of virtual simulation, we will see more innovative uses of this technology in the future, which may be the basic way for us to communicate and work.

\section{Data Availability}

No data were used to support this study.

\section{Conflicts of Interest}

The author declares that there are no conflicts of interest regarding the publication of this article.

\section{References}

[1] P. Rosedale, "Virtual reality: the next disruptor: a new kind of worldwide communication," IEEE Consumer Electronics Magazine, vol. 6, no. 1, pp. 48-50, 2016.

[2] E. Bastug, M. Bennis, M. Medard, and M. Debbah, "Toward interconnected virtual reality: opportunities, challenges, and enablers," IEEE Communications Magazine, vol. 55, no. 6, pp. 110-117, 2017.

[3] G. Riva, M. Bacchetta, and M. Baruffi, "Virtual reality environment for body image modification: a multidimensional therapy for the treatment of body image in obesity and related pathologies," CyberPsychology and Behavior, vol. 3, no. 3, pp. 421-431, 2016.

[4] J. Lenoir, S. Cotin, and C. Duriez, "Workshop on virtual reality interaction and physical simulation," in Journal of Preventive Medicine Information, F. Ganovelli and C. Mendoza, Eds., vol. 61, no. 13, pp. 2132-2141, 2017.

[5] K. S. Hsu, J. F. Jiang, and H. Y. Wei, "Application of the environmental sensation learning vehicle simulation platform in virtual reality," Eurasia Journal of Mathematics, Science and Technology Education, vol. 12, no. 5, pp. 1477-1485, 2016.

[6] C. T. Chen, S. Y. Chen, and C. H. Liao, "AN interactive nanomanipulation visualization based $\mathrm{ON}$ molecular dynamics simulation and virtual reality," Transactions of the Canadian Society for Mechanical Engineering, vol. 37, no. 3, pp. 991-1000, 2016.

[7] V. Khanduja, J. E. Lawrence, and E. Audenaert, “Testing the construct validity of a virtual reality hip arthroscopy simulator," Arthroscopy: The Journal of Arthroscopic \& Related Surgery, vol. 3, no. 3, pp. 566-571, 2016.

[8] S. Y. Hsu, T. Y. Fang, and S. C. Yeh, "Three-dimensional, virtual reality vestibular rehabilitation for chronic imbalance problem caused by Ménière's disease: a pilot study," Disability \& Rehabilitation, vol. 39, no. 16, pp. 1-6, 2016.

[9] C. Huang, S. K. Agrawal, and H. M. Ladak, "Virtual reality simulator for training in myringotomy with tube placement," Journal of Medical and Biological Engineering, vol. 36, no. 2, pp. 214-225, 2016.

[10] L. R. Valmaggia, F. Day, and M. Rus-Calafell, "Using virtual reality to investigate psychological processes and mechanisms associated with the onset and maintenance of psychosis: a systematic review," Social Psychiatry and Psychiatric Epidemiology, vol. 51, no. 7, pp. 921-936, 2016.

[11] J. Shin, G. An, and J. S. Park, "Application of precise indoor position tracking to immersive virtual reality with translational movement support," Multimedia Tools and Applications, vol. 75, no. 20, pp. 1-20, 2016.

[12] M. Y. Jeng, F. Y. Pai, and T. M. Yeh, "The virtual reality leisure activities experience on elderly people," Applied Research in Quality of Life, vol. 12, no. 1, pp. 1-17, 2016.

[13] A. S. S. Thomsen, D. Bach-Holm, H. Kjærbo et al., "Operating room performance improves after proficiency-based virtual reality cataract surgery training," Ophthalmology, vol. 124, no. 4 , pp. 524-531, 2017.

[14] A. Yasser and Noureldin, "Is there a place for virtual reality simulators in assessment of competency in percutaneous renal access?" World Journal of Urology, vol. 34, no. 5, pp. 733-739, 2016.

[15] J. Good, S. Parsons, N. Yuill, and M. Brosnan, "Virtual reality and robots for autism: moving beyond the screen," Journal of Assistive Technologies, vol. 10, no. 4, pp. 211-216, 2016.

[16] F. Ozkan, "Introducing VR first: unlocking the door to a future in virtual reality [future directions]," IEEE Consumer Electronics Magazine, vol. 5, no. 4, pp. 25-26, 2016.

[17] M. I. R. Severiano, B. S. Zeigelboim, H. A. G. Teive, G. J. B. Santos, and V. R. Fonseca, "Effect of virtual reality in Parkinson's disease: a prospective observational study," Arquivos de Neuro-Psiquiatria, vol. 76, no. 2, pp. 78-84, 2018.

[18] M. Coxon, N. Kelly, and S. Page, "Individual differences in virtual reality: are spatial presence and spatial ability linked?" Virtual Reality, vol. 20, no. 4, pp. 203-212, 2016.

[19] A. P. R. Johnston, J. Rae, N. Ariotti et al., "Journey to the centre of the cell: virtual reality immersion into scientific data," Traffic, vol. 19, no. 2, pp. 105-110, 2018.

[20] I. Bergstrom, S. Azevedo, P. Papiotis, N. Saldanha, and M. Slater, "The plausibility of a string quartet performance in virtual reality," IEEE Transactions on Visualization and Computer Graphics, vol. 23, no. 4, pp. 1352-1359, 2017.

[21] "Supporting skill acquisition in cochlear implant surgery through virtual reality simulation," Cochlear Implants International, vol. 18, no. 2, pp. 89-96, 2017. 